Bull. Chem. Soc. Ethiop. 2005, 19(2), 233-241.

Printed in Ethiopia

ISSN 1011-3924

(c) 2005 Chemical Society of Ethiopia

\title{
SYNTHESIS OF 2,3-UNSATURATED FURANIC HEX- AND PENT-ENOPYRANOSIDE EMPLOYING THE FERRIER REARRANGEMENT
}

\author{
Soro Yaya ${ }^{\mathrm{a}^{*}}$, Siaka Sorho $^{\mathrm{a}}$, Louis Cottier $^{\mathrm{b}}$ and Gérard Descotes ${ }^{\mathrm{b}}$ \\ aa Laboratoire des Procédés Industriels de Synthèse et d'Environement, Institut National \\ Polytechnique Félix Houphouët-Boigny, BP 991 Yamoussoukro, Côte d’Ivoire \\ ${ }^{\mathrm{b}}$ Laboratoire de Chimie Organique 2, UMR CNRS 5622, Université Claude Bernard Lyon 1, 43 \\ Boulevard du 11 Novembre 1918, 69622-Villeurbanne, France
}

(Received January 28, 2005; revised March 22, 2005)

\begin{abstract}
Glycosidation of various glycals with furanic alcohols in presence of catalytic amount of ceric(IV) ammonium nitrate under neutral condition or using Lewis acid-catalysed proceeds smoothly to afford the corresponding 2,3-unsaturated glycosides in good yields. In the hexose series predominantly $\alpha$-D-anomers resulted while $\beta$-D-anomers are predominant in the pentose serie.
\end{abstract}

KEY WORDS: Ferrier rearrangement, Furanic alcohols, Glycosides, Ceric(IV) ammonium nitrate, Lewis acid catalyst

\section{INTRODUCTION}

Glycals, 1,2-unsaturated derivatives of hexoses and pentoses, are among the most versatile chiral building blocks. Not surprisingly, glycals have been the subject of considerable interest in carbohydrate chemistry [1], oligosaccharide synthesis [2], and the development of combinatorial synthesis of oligosaccharide libraries [3]. 2,3- or 3-desoxy sugar derived from 2,3-unsaturated glycosides are useful chiral intermediates in many bioactive molecules, such as antibiotics [4], possessing significant antitumor and antiviral activities [5, 6].

The acid-catalysed allylic rearrangement of glycals in presence of alcohols, known as the Ferrier rearrangement [7], is widely employed to obtain 2,3-unsaturated glycosides. The reaction, as originally stated by Ferrier, involves the formation of a cyclic allylic oxocarbenium ion intermediate to which the nucleophile adds, preferentially in a quasi-axial orientation.

In recent year, ceric(IV) ammonium nitrate (CAN) has attracted much attention as a catalyst in many carbon-carbon bond forming reactions [8]. It has also been widely used in carbonheteroatom bond formation and its use as catalyst in $O$-glycosidation reaction has been reported. Recently, Paul and Jayaraman described the formation of 2-deoxy-1-thioglycosides mediated with CAN [9]. The reaction may proceed through a one-electron transfer with initial formation of a radical cation and allylic oxonium intermediate [10].

In recent publication, we reported the glycosidation of 3,4,6-tri- $O$-acetyl-D-glucal 1 with six alcohols among which furanic alcohols $\mathbf{2 a}$ and $\mathbf{2 b}$ in presence of Lewis acid [11]. In our ongoing research on the glycosidation of glycals, we report here the glycosidation of 3,4,6-tri- $O$ acetyl-D-glucal 1 (Scheme 1) and 3,4-di-O-acetyl-D-xylal 4 (Scheme 2) with various furanic alcohols using ceric(IV) ammonium nitrate under neutral reaction conditions or Lewis acidcatalyst.

*Corresponding author. Dr SORO Yaya, BP 991 Yamoussoukro (Côte d’Ivoire).

E-mail: soro_y@yahoo.fr 


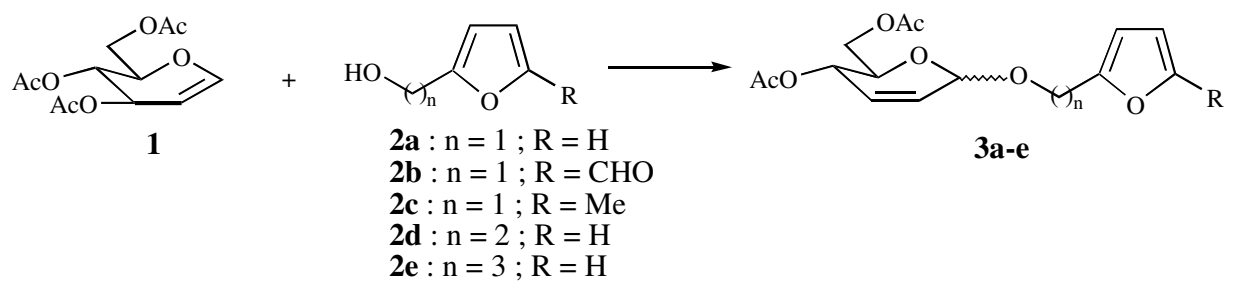

Scheme 1

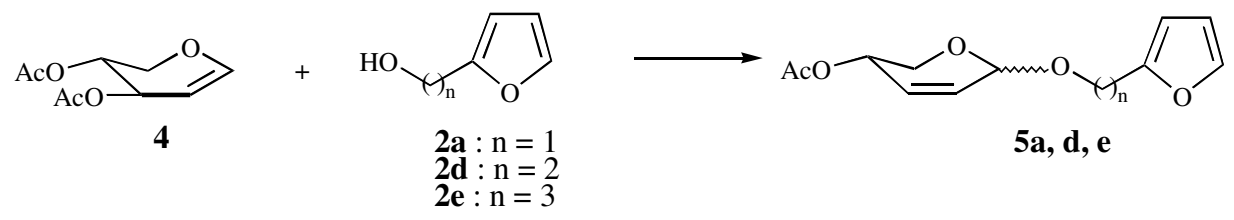

Scheme 2

To our knowledge, except $\mathbf{3 a}$ and $\mathbf{3 b}$, none of these glycosides have been reported in the literature.

\section{RESULTS AND DISCUSSION}

Starting furanic alcohols $\mathbf{2 c}, \mathbf{2 d}$ and $\mathbf{2 e}$ were respectively prepared with sodium borohydride $\left(\mathrm{NaBH}_{4}\right)$ of 5-methyl-2-hydroxymethylfurfural, by addition of furyllithium on ethylene oxide [12] and reduction in two steps of 3-(2-furyl)-acrolein[13] in good yields.

The reaction of alcohols 2a-e with glucal 1, carried out in presence of boron trifluoride [7] (method A), ferric chloride [11] (method B) and CAN [10] (method C), afforded the corresponding 2,3-unsaturated glucopyranosides 3a-e (Table 1).

Table 1. Glycosidation of glucal 1 with furanic alcohols 2a-e.

\begin{tabular}{|c|c|c|c|c|}
\hline \multirow{2}{*}{ Entry } & \multirow{3}{*}{ Alcohol } & \multicolumn{3}{|c|}{ Product 3a-e } \\
\cline { 3 - 5 } & Yield \% $(\alpha / \beta)$ & $\begin{array}{c}\text { Method B } \\
\text { Yield } \%(\alpha / \beta)\end{array}$ & $\begin{array}{c}\text { Method C } \\
\text { Yield \% }(\alpha / \beta)\end{array}$ \\
\hline 1 & 2a & $27(81 / 19)$ & $54(75 / 25)$ & $70(71 / 30)$ \\
2 & 2b & $86(87 / 13)$ & $82(88 / 12)$ & $90(84 / 16)$ \\
3 & 2c & degradation & degradation & degradation \\
4 & 2d & $65(88 / 12)$ & $45(92 / 8)$ & --- \\
5 & 2e & $87(92 / 8)$ & $59(94 / 6)$ & --- \\
\hline
\end{tabular}

Method $\mathrm{A}: \mathrm{BF}_{3} \cdot \mathrm{Et}_{2} \mathrm{O}$ in dichloromethane, $0{ }^{\circ} \mathrm{C}$ and room temperature, 24 hours.

Method $\mathrm{B}: \mathrm{FeCl}_{3}$ in chlorobenzene, room temperature, 24 hours.

Method C: CAN in acetonitrile, room temperature, 24 hours.

With boron trifluoride as the catalyst (method A), the yields from monosubstitued furanic alcohols 2a, 2d and 2e increased with the lentgh of carbon chain while the yields were lower than those of method A in presence of ferric chloride (method B). From hydroxymethylfuran 2a, the best yield was obtain using CAN as catalyst.

Bull. Chem. Soc. Ethiop. 2005, 19(2) 
Introduction of a methyl group in position 5 of furanic alcohol $\mathbf{2 a}$ led to polymeric compounds with all catalyst used (entry 3) whereas the presence of aldehyde group led to product $\mathbf{3 b}$ in good yields in all methods (entry 2). This difference could be explained by the competition between the formation of cyclic allylic oxocarbenium ion and polarisation of the hydroxy group. In fact, the presence of methyl group donnor increase the basicity of oxygen of hydroxy group whereas a formyl group decrease it. If oxygen is more basic, the degradation of furanic alcohols occur.

In all cases, compounds 3 were obtained as a mixture of $\alpha$ - and $\beta$-anomers. The selectivity $\alpha / \beta$ was small influenced by the catalyst used and was in favor of $\alpha$-anomers. The amount of $\alpha$ anomers ranged from 70 to $94 \%$ according to the catalyst employed and was calculated from ${ }^{13} \mathrm{C}$ NMR spectra [11]. The mixture was separated by chromatography column under deacetylated form and each anomer was reacetylated to produce $\mathbf{3} \alpha$ and $\mathbf{3} \beta$.

To explain the major formation of $\alpha$-anomers, Shostakovskii et al.[14] suggested that the reaction should be proceeded by a mechanism involving the anchimeric assistance of the acetate group at C-6 on the anomeric center. This assistance involved an allylic rearrangement reaction and departure of acetoxy group at $\mathrm{C}-3$, followed by the nucleophilic attack at $\mathrm{C}-1$ by the lower face to give 2,3-unsaturated glycosides. The $\alpha / \beta$ anomers selectivity was also enhanced with the length of carbon chain.

In pentose series, the similar reactions have been done with 3,4-di- $O$-acetyl-D-xylal 4 using only CAN as catalyst regarding the best yields obtained in hexose series (Scheme 2).

The treatment of xylal $\mathbf{4}$ with monosubstitued furanic alcohols $2 \mathbf{2 a}, \mathbf{2 d}$ and $\mathbf{2 e}$ in presence of $20 \mathrm{mmol} \%$ of CAN in acetonitrile at room temperature gave the corresponding 2,3-unsaturated glycopyranoside 5a, 5d and 5e with high $\beta$-anomers selectivity (Table 2).

Table 2. Glycosidation of xylal $\mathbf{4}$ with furanic alcohols $\mathbf{2 a}, \mathbf{2 d}$ and $\mathbf{2 e}$.

\begin{tabular}{|cccc|}
\hline Entry & Alcohol & Product & Yield $(\%)(\alpha / \beta)$ \\
\hline 1 & 2a & 5a & $94(22 / 78)$ \\
2 & 2d & 5d & $69(18 / 82)$ \\
3 & 2e & 5e & $43(25 / 75)$ \\
\hline
\end{tabular}

The yields of reactions decrease when the lentgh of carbon chain increase. Polymeric product was obtained with disubstitued furanic alcohol $\mathbf{2 c}$.

The structures of $\alpha$ - and $\beta$-anomers, easily separated on chromatography column, were established by ${ }^{13} \mathrm{C}$ and ${ }^{1} \mathrm{H}$ NMR spectra. As mentionned by Doboszewski et al. [15], the $\alpha-$ anomer adopted the ${ }^{0} \mathrm{H}_{5}$ conformation with large vicinal coupling between $\mathrm{H}-4$ and both $\mathrm{H}-5 \mathrm{a}$ and $\mathrm{H}-5 \mathrm{e}$ whereas $\beta$-anomers adopted conformation ${ }^{5} \mathrm{H}_{0}$ (Figure 1) with small coupling constants.<smiles>CCOC1COC2CC1C2OC(C)=O</smiles>

Conformation ${ }^{0} \mathrm{H}_{5}(\alpha$-anomer)

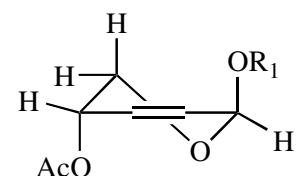

Conformation ${ }^{5} \mathrm{H}_{0}(\beta$-anomer $)$

Figure 1. The structures of $\alpha$ - and $\beta$-anomers.

In this series, the position of the H-4 signals of the two anomers differed considerably. For $\alpha$-anomers, this signal appeared at about $5.28 \mathrm{ppm}$, whereas for $\beta$-anomers it appeared at about 
$4.93 \mathrm{ppm}$ (table 3 ). The $\beta$-anomers selectivity is in accord with previous findings in the pentose series [16], which was justified by the missing influence of the large substituted 5acetoxymethyl group.

Table 3. Coupling constants values between H-4 and H-5 and chemical shifts of H-4 in ${ }^{1} \mathrm{H}$ NMR (200 $\mathrm{MHz}, \mathrm{CDCl}_{3}$ ) of compounds 5 .

\begin{tabular}{|c|c|c|l|}
\hline Compound & $\mathrm{J}_{4,5 \mathrm{a}}(\mathrm{Hz})$ & $\mathrm{J}_{4,5 \mathrm{e}}(\mathrm{Hz})$ & $\delta \mathrm{H}-4$ in ppm (multiplicity) \\
\hline 5a $\alpha$ & 9.8 & 2.0 & $5.32(\mathrm{ddd})$ \\
$\beta$ & 2.8 & 0 & $4.96(\mathrm{t})$ \\
& & & \\
5d $\alpha$ & 5.8 & 1.7 & $5.28(\mathrm{ddd})$ \\
$\beta$ & 2.7 & 1.7 & $4.93(\mathrm{dd})$ \\
& & & \\
5e $\alpha$ & 7.7 & 2.1 & $5.28(\mathrm{ddd})$ \\
$\beta$ & 2.8 & 0 & $4.93(\mathrm{~m})$ \\
\hline
\end{tabular}

\section{CONCLUSION}

Many furanic hex- and pent-enopyranosides have been prepared via the Ferrier rearrangement. With glucal, the presence of a methyl group at C-5 on the furanic alcohol led to polymeric compounds whereas a formyl group favoured the reaction. In hexose series, when using boron trifluoride, the yields enhanced with the length of carbon chain with $\alpha$-anomers selectitivity whereas in pentose series, use of ceric(IV) ammonium nitrate produced contrary results with $\beta$ anomers selectivity.

\section{EXPERIMENTAL}

The 200 or $300 \mathrm{MHz}{ }^{1} \mathrm{H}$ NMR and 50 or $75 \mathrm{MHz}{ }^{13} \mathrm{C}$ NMR spectra were recorded with a Bruker AC spectrometer in $\mathrm{CDCl}_{3}$ with TMS as an internal standard. Elemental analyses were performed in the CNRS Analysis Department of Solaize (France). Optical rotations were measured with a Perkin-Elmer 241 polarimeter in Villeurbanne (France). Thin-layer chromatography (TLC) was carried out on plates coated with silica gel $60(40-63 \mu \mathrm{m})$ followed by spraying the plates with dilute sulfuric acid (25\%). The mass spectroscopy in chemical ionization mode were performed on a Finnigan Mat 95 XL spectrometer in Villeurbanne (France). Starting compound 1 was bought from Aldrich.

General methods for the preparation of 2,3-unsaturated glycopyranosides $\mathbf{3}$ and $\mathbf{5}$

Method A. 3,4,6-tri- $O$-acetyl-D-glucal 1 (503 mg, $1.85 \mathrm{mmol}$ ) and an appropriate furanic alcohol $2(4 \mathrm{mmol}, 2.16$ equiv. $)$ in dry dichloromethane $(10 \mathrm{~mL})$ were stirred in a $50 \mathrm{~mL}$ round-bottom flask under nitrogen atmosphere. Boron trifluoride etherate $(24 \mu \mathrm{L}, 0.2 \mathrm{mmol}, 5 \mathrm{mmol} \%)$ was added to it at $0{ }^{\circ} \mathrm{C}$ and the stirring continued for 24 hours at room temperature. Thin-layer chromatography $\left(\mathrm{CH}_{2} \mathrm{Cl}_{2} / \mathrm{AcOEt} 5 / 0.5 \mathrm{v} / \mathrm{v}\right)$ showed the disappearance of the starting material $\mathbf{1}$. The mixture was neutralized by addition of a satured solution of $\mathrm{NaHCO}_{3}$ and the organic layer was extracted with dichloromethane $(3 \times 10 \mathrm{~mL})$. The organic layer was washed with water, then brine, dried on $\mathrm{MgSO}_{4}$ and concentrated. The raw product was purified by silica gel column $\left(\mathrm{CH}_{2} \mathrm{Cl}_{2} / \mathrm{AcOEt} 5 / 0.5 \mathrm{v} / \mathrm{v}\right)$ to give a mixture of $\alpha$ - and $\beta$-anomers 3 . The yields are reported in Table 1. 
Method B. To a mixture of 3,4,6-tri- $O$-acetyl-D-glucal 1 (272 $\mathrm{mg}, 1 \mathrm{mmol})$ and an appropriate furanic alcohol 2 (4 mmol, 4 equiv.) in dry chlorobenzene $(12 \mathrm{~mL}$ ) was added ferric chloride $(162 \mathrm{mg}, 1 \mathrm{mmol})$ at room temperature. The mixture was stirred in a $50 \mathrm{~mL}$ round-bottom flask under nitrogen atmosphere and the reaction followed by thin-layer chromatography $\left(\mathrm{CH}_{2} \mathrm{Cl}_{2} /\right.$ AcOEt 5/0.5 v/v). After filtration on celite and neutralisation with aqueous $\mathrm{NaHCO}_{3}$, the aqueous layer was extracted with dichloromethane $(3 \mathrm{x} 10 \mathrm{~mL})$. The organic layer was washed with water, then brine, dried on $\mathrm{MgSO}_{4}$ and concentrated. The raw product was purified by silica gel column $\left(\mathrm{CH}_{2} \mathrm{Cl}_{2} / \mathrm{AcOEt} 5 / 0.5 \mathrm{v} / \mathrm{v}\right)$ to give a mixture of $\alpha$ - and $\beta$-anomers 3 . The yields are reported in Table 1.

Method $C$. A mixture of 3,4-di- $O$-acetyl-D-xylal $4(600 \mathrm{mg}, 3 \mathrm{mmol})$, an appropriate furanic alcohol 2 (4.5 mmol, 1.5 equiv.) and ceric(IV) ammonium nitrate (330 mg, $20 \mathrm{mmol} \%$ ) in acetonitrile $(9 \mathrm{~mL})$ was stirred at room temperature in a $50 \mathrm{~mL}$ round-bottom flask under nitrogen atmosphere and the reaction followed by thin-layer chromatography $\left(\mathrm{CH}_{2} \mathrm{Cl}_{2} / \mathrm{AcOEt}\right.$ $5 / 0.5 \mathrm{v} / \mathrm{v})$. After the disappearance of the xylal (24 hours), the mixture was quenched with water $(5 \mathrm{~mL})$ and extracted with ethyl acetate $(3 \times 10 \mathrm{~mL})$. The combined organic layers were dried over anhydrous $\mathrm{MgSO}_{4}$, concentred in vacuo and purified by column chromatography on silica gel (petroleum ether/ethyl acetate $5 / 2 \mathrm{v} / \mathrm{v}$ ) to afford pure $\alpha$ - and $\beta$-anomers $\mathbf{5}$. The yields are reported in Table 2.

Furfuryl 4,6-di-O-acetyl-2,3-dideoxy- $\alpha$-D-erythro-hex-2-enopyranoside $3 \boldsymbol{a} \alpha$. Compound 3a $\alpha$ was obtained as a light yellow oil. The specific rotation of pure material is $[\alpha]_{\mathrm{D}}{ }^{20}-40^{\circ}$ (c 1 , $\mathrm{CHCl}_{3}$ ); $\mathrm{R}_{\mathrm{f}}=0.37$ (dichloromethane/ethyl acetate 50/0.5 v/v). ${ }^{1} \mathrm{H}$ NMR $(300 \mathrm{MHz}$, $\left.\mathrm{CDCl}_{3}+\mathrm{TMS}\right): \delta 7.44\left(\mathrm{t}, 1 \mathrm{H}, \mathrm{J}_{11,9}=\mathrm{J}_{11,10}=1.3 \mathrm{~Hz}, \mathrm{H}-11\right), 6.37\left(\mathrm{~d}, 1 \mathrm{H}, \mathrm{J}_{10,9}=1.3 \mathrm{~Hz}, \mathrm{H}-10\right), 6.36$ $\left(\mathrm{d}, 1 \mathrm{H}, \mathrm{J}_{9,10}=1.3 \mathrm{~Hz}, \mathrm{H}-9\right), 5.88$ (ddd, $\left.1 \mathrm{H}, \mathrm{J}_{2,1}=0.7 \mathrm{~Hz}, \mathrm{~J}_{2,4}=1.8 \mathrm{~Hz}, \mathrm{~J}_{2,3}=10.6 \mathrm{~Hz}, \mathrm{H}-2\right), 5.85$ $\left(\mathrm{ddd}, 1 \mathrm{H}, \mathrm{J}_{3,1}=0.7 \mathrm{~Hz}, \mathrm{~J}_{3,4}=1.8 \mathrm{~Hz}, \mathrm{~J}_{3,2}=10.6 \mathrm{~Hz}, \mathrm{H}-3\right), 5.35\left(\mathrm{ddd}, 1 \mathrm{H}, \mathrm{J}_{4,2}=1.8 \mathrm{~Hz}, \mathrm{~J}_{4,3}=1.8\right.$ $\left.\mathrm{Hz}, \mathrm{J}_{4,5}=9.7 \mathrm{~Hz}, \mathrm{H}-4\right), 5.16\left(\mathrm{t}, 1 \mathrm{H}, \mathrm{J}_{1,2}=\mathrm{J}_{1,3}=0.7 \mathrm{~Hz}, \mathrm{H}-1\right), 4.71\left(\mathrm{~d}, 1 \mathrm{H}, \mathrm{J}_{7 \mathrm{a}, 7 \mathrm{~b}}=12.7 \mathrm{~Hz}, \mathrm{H}-7 \mathrm{a}\right)$, $4.59\left(\mathrm{~d}, 1 \mathrm{H}, \mathrm{J}_{7 \mathrm{~b}, 7 \mathrm{a}}=12.7 \mathrm{~Hz}, \mathrm{H}-7 \mathrm{~b}\right), 4.28\left(\mathrm{dd}, 1 \mathrm{H}, \mathrm{J}_{6 \mathrm{a}, 5}=5.3 \mathrm{~Hz}, \mathrm{~J}_{6 \mathrm{a}, 6 \mathrm{~b}}=11 \mathrm{~Hz}, \mathrm{H}-6 \mathrm{a}\right), 4.18$ (dd, $\left.1 \mathrm{H}, \mathrm{J}_{6 \mathrm{~b}, 5}=2.4 \mathrm{~Hz}, \mathrm{~J}_{6 \mathrm{~b}, 6 \mathrm{a}}=11 \mathrm{~Hz}, \mathrm{H}-6 \mathrm{~b}\right), 4.14(\mathrm{~m}, 1 \mathrm{H}, \mathrm{H}-5), 2.15$ (s, 3H, OAc), 2.09 (s, 3H, OAc). ${ }^{13} \mathrm{C}$ NMR (50 MHz, CDCl + TMS): $\delta 170.3\left(\mathrm{CO}_{2}\right), 170.3\left(\mathrm{CO}_{2}\right), 151.1(\mathrm{C}-8), 143.1(\mathrm{C}-$ 11), 129.5 (C-2), 127.6 (C-3), 126.0 (C-10), 110.4 (C-9), 93.3 (C-1), 67.1 (C-5), 65.2 (C-4), 62.9 (C-6), $61.8(\mathrm{C}-7), 21.0\left(\mathrm{CH}_{3}\right), 20.9\left(\mathrm{CH}_{3}\right)$. Anal. calcd for $\mathrm{C}_{15} \mathrm{H}_{18} \mathrm{O}_{7}(310.29)$ : C, 58.06; $\mathrm{H}$, $5.85 \%$. Found: C, $58.06 ; \mathrm{H}, 5.67 \%$.

Furfuryl 4,6-di-O-acetyl-2,3-dideoxy- $\beta$-D-erythro-hex-2-enopyranoside $3 \mathbf{a} \beta$. Compound 3a $\beta$ was obtained as a light yellow oil. The specific rotation of pure material is $[\alpha]_{\mathrm{D}}{ }^{20}+81^{\circ}(\mathrm{c} 0.45$, $\left.\mathrm{CHCl}_{3}\right) ; \mathrm{R}_{\mathrm{f}}=0.26$ (dichloromethane/ethyl acetate 50/0.5 v/v). ${ }^{1} \mathrm{H}$ NMR $(300 \mathrm{MHz}$, $\left.\mathrm{CDCl}_{3}+\mathrm{TMS}\right): \delta 7.43\left(\mathrm{t}, 1 \mathrm{H}, \mathrm{J}_{11,9}=\mathrm{J}_{11,10}=1.3 \mathrm{~Hz}, \mathrm{H}-11\right), 6.36\left(\mathrm{~d}, 2 \mathrm{H}, \mathrm{J}_{10,9}=1.3 \mathrm{~Hz}, \mathrm{~J}_{9,10}=1.3\right.$ $\mathrm{Hz}, \mathrm{H}-9$ and $\mathrm{H}-10$ ), 5.91 (ddd, $1 \mathrm{H}, \mathrm{J}_{2,1}=1.1 \mathrm{~Hz}, \mathrm{~J}_{2,3}=10.3 \mathrm{~Hz}, \mathrm{~J}_{2,4}=1.4 \mathrm{~Hz}, \mathrm{H}-2$ ), 5.85 (ddd, $\left.1 \mathrm{H}, \mathrm{J}_{3,1}=0.8 \mathrm{~Hz}, \mathrm{~J}_{3,2}=10.3 \mathrm{~Hz}, \mathrm{~J}_{3,4}=1.7 \mathrm{~Hz}, \mathrm{H}-3\right), 5.44\left(\mathrm{ddd}, 1 \mathrm{H}, \mathrm{J}_{4,2}=1.4 \mathrm{~Hz}, \mathrm{~J}_{4,3}=1.7 \mathrm{~Hz}\right.$, $\left.\mathrm{J}_{4,5}=9.6 \mathrm{~Hz}, \mathrm{H}-4\right), 5.13$ (s, br, 1H, H-1), $4.72\left(\mathrm{~d}, 1 \mathrm{H}, \mathrm{J}_{7 \mathrm{a}, 7 \mathrm{~b}}=12.7 \mathrm{~Hz}, \mathrm{H}-7 \mathrm{a}\right), 4.60\left(\mathrm{~d}, 1 \mathrm{H}, \mathrm{J}_{7 \mathrm{~b}, 7 \mathrm{a}}=\right.$ $12.7 \mathrm{~Hz}, \mathrm{H}-7 \mathrm{~b}), 4.27$ (dd, 1H, J $\left.\mathrm{J}_{6,5}=4.7 \mathrm{~Hz}, \mathrm{~J}_{6 \mathrm{a}, 6 \mathrm{~b}}=9.4 \mathrm{~Hz}, \mathrm{H}-6 \mathrm{a}\right), 4.23\left(\mathrm{dd}, 1 \mathrm{H}, \mathrm{J}_{6 \mathrm{~b}, 5}=2.4 \mathrm{~Hz}\right.$, $\left.\mathrm{J}_{6 \mathrm{~b}, 6 \mathrm{a}}=9.4 \mathrm{~Hz}, \mathrm{H}-6 \mathrm{~b}\right), 4.12(\mathrm{~m}, 1 \mathrm{H}, \mathrm{H}-5), 2.14$ (s, 3H, OAc), 2.06 (s, 3H, OAc). ${ }^{13} \mathrm{C}$ NMR (50 $\left.\mathrm{MHz}, \mathrm{CDCl}_{3}+\mathrm{TMS}\right): \delta 170.8\left(\mathrm{CO}_{2}\right), 170.7\left(\mathrm{CO}_{2}\right), 150.9$ (C-8), 143.1 (C-11), $130.3(\mathrm{C}-2), 127.6$ (C-3), 125.9 (C-10), 109.7 (C-9), 93.3 (C-1), 72.9 (C-5), 64.2 (C-4), 63.5 (C-6), 61.2 (C-7), 20.8 $\left(\mathrm{CH}_{3}\right), 20.8\left(\mathrm{CH}_{3}\right)$. Anal. calcd for $\mathrm{C}_{15} \mathrm{H}_{18} \mathrm{O}_{7}(\mathrm{M}=310.29)$ : C, 58.06; H, 5.85\%. Found: $\mathrm{C}$, 58.02 ; H, 5.78\%.

Furfuraldehyde-5'-methyl 4,6-di-O-acetyl-2,3-dideoxy- $\alpha$-D-erythro-hex-2-enopyranoside $3 \boldsymbol{b} \alpha$. Compound $\mathbf{3 b} \alpha$ was obtained as a yellow oil. The specific rotation of pure material is $[\alpha]_{\mathrm{D}}{ }^{20}$ 
$+73^{\circ}\left(\mathrm{c} 1, \mathrm{CHCl}_{3}\right) ; \mathrm{R}_{\mathrm{f}}=0.32$ (dichloromethane/ethyl acetate $\left.5 / 0.5 \mathrm{v} / \mathrm{v}\right) .{ }^{1} \mathrm{H}$ NMR $(300 \mathrm{MHz}$, $\mathrm{CDCl}_{3}+\mathrm{TMS}$ ): $\delta 9.61$ (s, 1H, CHO), $7.2\left(\mathrm{~d}, 1 \mathrm{H}, \mathrm{J}_{10,9}=3.5 \mathrm{~Hz}, \mathrm{H}-10\right), 6.54\left(\mathrm{~d}, 1 \mathrm{H}, \mathrm{J}_{9,10}=3.5 \mathrm{~Hz}\right.$, $\mathrm{H}-9$ ), $5.96\left(\mathrm{ddd}, 1 \mathrm{H}, \mathrm{J}_{2,1}=1 \mathrm{~Hz}, \mathrm{~J}_{2,3}=11 \mathrm{~Hz}, \mathrm{~J}_{2,4}=1 \mathrm{~Hz}, \mathrm{H}-2\right), 5.84\left(\mathrm{ddd}, 1 \mathrm{H}, \mathrm{J}_{3,1}=1.5 \mathrm{~Hz}, \mathrm{~J}_{3,2}\right.$ $\left.=11 \mathrm{~Hz}, \mathrm{~J}_{3,4}=1.6 \mathrm{~Hz}, \mathrm{H}-3\right), 5.32\left(\mathrm{ddd}, 1 \mathrm{H}, \mathrm{J}_{4,2}=1 \mathrm{~Hz}, \mathrm{~J}_{4,3}=1.6 \mathrm{~Hz}, \mathrm{~J}_{4,5}=4.87 \mathrm{~Hz}, \mathrm{H}-4\right), 5.14$ $\left(\mathrm{dd}, 1 \mathrm{H}, \mathrm{J}_{1,2}=1 \mathrm{~Hz}, \mathrm{~J}_{1,3}=1.5 \mathrm{~Hz}, \mathrm{H}-1\right), 4.77\left(\mathrm{~d}, 1 \mathrm{H}, \mathrm{J}_{7 \mathrm{a}, 7 \mathrm{~b}}=13.3 \mathrm{~Hz}, \mathrm{H}-7 \mathrm{a}\right), 4.64\left(\mathrm{~d}, 1 \mathrm{H}, \mathrm{J}_{7 \mathrm{~b}, 7 \mathrm{a}}=\right.$ $13.3 \mathrm{~Hz}, \mathrm{H}-7 \mathrm{~b}$ ), 4.23 (dd, 1H, $\left.\mathrm{J}_{6 \mathrm{a}, 5}=4.9 \mathrm{~Hz}, \mathrm{~J}_{6 \mathrm{a}, 6 \mathrm{~b}}=9.9 \mathrm{~Hz}, \mathrm{H}-6 \mathrm{a}\right), 4.18$ (dd, 1H, $\mathrm{J}_{6 \mathrm{~b}, 5}=2.5 \mathrm{~Hz}$, $\left.\mathrm{J}_{6 \mathrm{~b}, 6 \mathrm{a}}=9.9 \mathrm{~Hz}, \mathrm{H}-6 \mathrm{~b}\right), 4.1$ (m, 1H, H-5), 2.08 (s, 3H, OAc), 2.05 (s, 3H, OAc). ${ }^{13} \mathrm{C}$ NMR (50 $\left.\mathrm{MHz}, \mathrm{CDCl}_{3}+\mathrm{TMS}\right): \delta 178.2(\mathrm{CHO}), 171.1\left(\mathrm{CO}_{2}\right), 170.7\left(\mathrm{CO}_{2}\right), 157.8(\mathrm{C}-8), 153.2(\mathrm{C}-11)$, 130.3 (C-2), 127.4 (C-3), 122.4 (C-10), 112.1 (C-9), 94.4 (C-1), 67.6 (C-5), 65.5 (C-4), 63.1 (C6), $62.4(\mathrm{C}-7), 21.3\left(\mathrm{CH}_{3}\right), 21.3\left(\mathrm{CH}_{3}\right)$. Anal. calcd for $\mathrm{C}_{16} \mathrm{H}_{18} \mathrm{O}_{8}(\mathrm{M}=338.30): \mathrm{C}, 56.80 ; \mathrm{H}$, $5.36 \%$. Found: C, $56.65 ; \mathrm{H}, 5.20 \%$.

Furfuraldehyde-5'-methyl 4,6-di-O-acetyl-2,3-dideoxy- $\beta$-D-erythro-hex-2-enopyranoside $3 \boldsymbol{b} \beta$. Compound 3b $\beta$ was obtained as a yellow oil. The specific rotation of pure material is $\alpha_{\mathrm{D}}{ }^{20}+74^{\circ}\left(\mathrm{c} 1, \mathrm{CHCl}_{3}\right) ; \mathrm{R}_{\mathrm{f}}=0.32$ (dichloromethane/ethyl acetate $\left.5 / 0.5 \mathrm{v} / \mathrm{v}\right) .{ }^{1} \mathrm{H} \mathrm{NMR}(300 \mathrm{MHz}$, $\mathrm{CDCl}_{3}+\mathrm{TMS}$ ): $\delta 9.60$ (s, $\left.1 \mathrm{H}, \mathrm{CHO}\right), 7.2\left(\mathrm{~d}, 1 \mathrm{H}, \mathrm{J}_{10,9}=3.5 \mathrm{~Hz}, \mathrm{H}-10\right), 6.54\left(\mathrm{~d}, 1 \mathrm{H}, \mathrm{J}_{9,10}=3.5 \mathrm{~Hz}\right.$, H-9), 6.01 (ddd, $\left.1 \mathrm{H}, \mathrm{J}_{2,1}=1.5 \mathrm{~Hz}, \mathrm{~J}_{2,4}=1.5 \mathrm{~Hz}, \mathrm{~J}_{2,3}=10.7 \mathrm{~Hz}, \mathrm{H}-2\right), 5.84\left(\mathrm{ddd}, 1 \mathrm{H}, \mathrm{J}_{3,1}=1.5\right.$ $\left.\mathrm{Hz}, \mathrm{J}_{3,4}=2 \mathrm{~Hz}, \mathrm{~J}_{3,2}=10.7 \mathrm{~Hz}, \mathrm{H}-3\right), 5.34(\mathrm{~m}, 1 \mathrm{H}, \mathrm{H}-4), 5.23\left(\mathrm{t}, 1 \mathrm{H}, \mathrm{J}_{1,2}=\mathrm{J}_{1,3}=1.5 \mathrm{~Hz}, \mathrm{H}-1\right)$, $4.83\left(\mathrm{~d}, 1 \mathrm{H}, \mathrm{J}_{7 \mathrm{a}, 7 \mathrm{~b}}=13.3 \mathrm{~Hz}, \mathrm{H}-7 \mathrm{a}\right), 4.66\left(\mathrm{~d}, 1 \mathrm{H}, \mathrm{J}_{7 \mathrm{~b}, 7 \mathrm{a}}=13.3 \mathrm{~Hz}, \mathrm{H}-7 \mathrm{~b}\right), 4.25\left(\mathrm{dd}, 1 \mathrm{H}, \mathrm{J}_{6 \mathrm{a}, 5}=4.9\right.$ $\left.\mathrm{Hz}, \mathrm{J}_{6 \mathrm{a}, 6 \mathrm{~b}}=9.9 \mathrm{~Hz}, \mathrm{H}-6 \mathrm{a}\right), 4.13\left(\mathrm{dd}, 1 \mathrm{H}, \mathrm{J}_{6 \mathrm{~b}, 5}=2.5 \mathrm{~Hz}, \mathrm{~J}_{6 \mathrm{~b}, 6 \mathrm{a}}=9.9 \mathrm{~Hz}, \mathrm{H}-6 \mathrm{~b}\right), 4.09$ (m, 1H, H-5), 2.08 (s, 3H, OAc), 2.05 (s, 3H, OAc). ${ }^{13} \mathrm{C}$ NMR (50 MHz, $\left.\mathrm{CDCl}_{3}+\mathrm{TMS}\right): \delta 178.1(\mathrm{CHO}), 171.1$ $\left(\mathrm{CO}_{2}\right), 170.7\left(\mathrm{CO}_{2}\right), 157.9$ (C-8), 153.1 (C-11), 130.0 (C-2), 127.0 (C-3), 122.4 (C-10), 112.0 (C-9), $94.5(\mathrm{C}-1), 73.3(\mathrm{C}-5), 64.3(\mathrm{C}-4), 63.7(\mathrm{C}-6), 61.8(\mathrm{C}-7), 21.2\left(\mathrm{CH}_{3}\right), 21.2\left(\mathrm{CH}_{3}\right)$. Anal. calcd for $\mathrm{C}_{16} \mathrm{H}_{18} \mathrm{O}_{8}(\mathrm{M}=338.30)$ : C, 56.80; H, 5.36\%. Found: $\mathrm{C}, 56.25 ; \mathrm{H}, 5.52 \%$.

Ethyl-(furyl-2')-2", 4,6-di-O-acetyl-2,3-dideoxy- $\alpha$-D-erythro-hex-2-enopyranoside $\quad 3 \boldsymbol{d} \quad \alpha$. Compound 3d $\alpha$ was obtained as a colourless oil. The specific rotation of pure material is $[\alpha]_{\mathrm{D}}{ }^{20}+77^{\circ}\left(\mathrm{c} 1, \mathrm{CHCl}_{3}\right) ; \mathrm{R}_{\mathrm{f}}=0.46\left(\right.$ dichloromethane/ethyl acetate 50/0.5 v/v). ${ }^{1} \mathrm{H}$ NMR $(300$ $\left.\mathrm{MHz}, \mathrm{CDCl}_{3}+\mathrm{TMS}\right): \delta 7.31\left(\mathrm{dd}, 1 \mathrm{H}, \mathrm{J}_{12,10}=0.8 \mathrm{~Hz}, \mathrm{~J}_{12,11}=1.8 \mathrm{~Hz}, \mathrm{H}-12\right), 6.3\left(\mathrm{dd}, 1 \mathrm{H}, \mathrm{J}_{11,12}=\right.$ $\left.1.8 \mathrm{~Hz}, \mathrm{~J}_{11,10}=3.1 \mathrm{~Hz}, \mathrm{H}-11\right), 6.09\left(\mathrm{dd}, 1 \mathrm{H}, \mathrm{J}_{10,12}=0.8 \mathrm{~Hz}, \mathrm{~J}_{10,11}=3.1 \mathrm{~Hz}, \mathrm{H}-10\right), 5.86(\mathrm{~d}, 1 \mathrm{H}$, $\mathrm{J}_{2,3}=10.5 \mathrm{~Hz}, \mathrm{H}-2$ ), 5.83 (ddd, $1 \mathrm{H}, \mathrm{J}_{3,4}=1.5 \mathrm{~Hz}, \mathrm{~J}_{3,1}=1.7 \mathrm{~Hz}, \mathrm{~J}_{3,2}=10.5 \mathrm{~Hz}, \mathrm{H}-3$ ), 5.27 (dd, $\left.1 \mathrm{H}, \mathrm{J}_{4,3}=1.5 \mathrm{~Hz}, \mathrm{~J}_{4,5}=3.3 \mathrm{~Hz}, \mathrm{H}-4\right), 5.03(\mathrm{~s}, \mathrm{br}, 1 \mathrm{H}, \mathrm{H}-1), 4.20\left(\mathrm{dd}, 1 \mathrm{H}, \mathrm{J}_{6 \mathrm{a}, 5}=5.3 \mathrm{~Hz}, \mathrm{~J}_{6 \mathrm{a}, 6 \mathrm{~b}}=\right.$ $12.1 \mathrm{~Hz}, \mathrm{H}-6 \mathrm{a}), 4.16$ (dd, 1H, J $\left.\mathrm{J}_{6 \mathrm{~b}, 5}=2.4 \mathrm{~Hz}, \mathrm{~J}_{6 \mathrm{~b}, 6 \mathrm{a}}=12.1 \mathrm{~Hz}, \mathrm{H}-6 \mathrm{~b}\right), 4.10(\mathrm{~m}, 1 \mathrm{H}, \mathrm{H}-5), 3.96$ (m, $1 \mathrm{H}, \mathrm{H}-7 \mathrm{a}), 3.81$ (ddd, $1 \mathrm{H}, \mathrm{J}_{7 \mathrm{~b}, 8 \mathrm{a}}=3.8 \mathrm{~Hz}, \mathrm{~J}_{7 \mathrm{~b}, 8 \mathrm{~b}}=6.8 \mathrm{~Hz}, \mathrm{~J}_{7 \mathrm{~b}, 7 \mathrm{a}}=16.5 \mathrm{~Hz}, \mathrm{H}-7 \mathrm{~b}$ ), 2.97 (t, 2H, $\mathrm{J}_{8,7 \mathrm{a}}$ $\left.=\mathrm{J}_{8,7 \mathrm{~b}}=6.8 \mathrm{~Hz}, 2 \mathrm{H}-8\right), 2.1$ (s, 3H, OAc), 2.07 (s, 3H, OAc). ${ }^{13} \mathrm{C} \mathrm{NMR}\left(50 \mathrm{MHz}, \mathrm{CDCl}_{3}+\mathrm{TMS}\right)$ : $\delta 170.7\left(\mathrm{CO}_{2}\right), 170.2\left(\mathrm{CO}_{2}\right), 152.8(\mathrm{C}-9), 141.2(\mathrm{C}-12), 110.3(\mathrm{C}-11), 106.0(\mathrm{C}-10), 129.2(\mathrm{C}-2)$, 127.7 (C-3), 94.5 (C-1), 67.0 (C-5), 66.9 (C-6), 65.3 (C-4), 63.0 (C-7), 28.9 (C-8), $20.9\left(\mathrm{CH}_{3}\right)$, $20.7\left(\mathrm{CH}_{3}\right)$. Anal. calcd for $\mathrm{C}_{16} \mathrm{H}_{20} \mathrm{O}_{7}(\mathrm{M}=324.32)$ : $\mathrm{C}, 59.25 ; \mathrm{H}, 6.22 \%$. Found: $\mathrm{C}, 59.10 ; \mathrm{H}$, $6.32 \%$.

Ethyl-(furyl-2')-2', 4,6-di-O-acetyl-2,3-dideoxy- $\beta$-D-erythro-hex-2-enopyranoside $\quad 3 \boldsymbol{d} \quad \beta$. Compound 3d $\beta$ was obtained as a colourless oil. The specific rotation of pure material is $[\alpha]_{\mathrm{D}}^{20}+116^{\mathrm{o}}\left(\mathrm{c} 1, \mathrm{CHCl}_{3}\right) ; \mathrm{R}_{\mathrm{f}}=0.46$ (dichloromethane/ethyl acetate 50/0.5 v/v). ${ }^{1} \mathrm{H}$ NMR $(300$ $\left.\mathrm{MHz}, \mathrm{CDCl}_{3}+\mathrm{TMS}\right): \delta 7.32\left(\mathrm{dd}, 1 \mathrm{H}, \mathrm{J}_{12,10}=0.8 \mathrm{~Hz}, \mathrm{~J}_{12,11}=1.8 \mathrm{~Hz}, \mathrm{H}-12\right), 6.28\left(\mathrm{dd}, 1 \mathrm{H}, \mathrm{J}_{11,12}=\right.$ $\left.1.8 \mathrm{~Hz}, \mathrm{~J}_{11,10}=3.1 \mathrm{~Hz}, \mathrm{H}-11\right), 6.07\left(\mathrm{dd}, 1 \mathrm{H}, \mathrm{J}_{10,12}=0.8 \mathrm{~Hz}, \mathrm{~J}_{10,11}=3.1 \mathrm{~Hz}, \mathrm{H}-10\right), 5.91(\mathrm{~d}, 1 \mathrm{H}$, $\left.\mathrm{J}_{2,3}=10.4 \mathrm{~Hz}, \mathrm{H}-2\right), 5.78$ (ddd, $1 \mathrm{H}, \mathrm{J}_{3,4}=1.3 \mathrm{~Hz}, \mathrm{~J}_{3,1}=1.7 \mathrm{~Hz}, \mathrm{~J}_{3,2}=10.4 \mathrm{~Hz}, \mathrm{H}-3$ ), 5.32 (dd, $\left.1 \mathrm{H}, \mathrm{J}_{4,3}=1.3 \mathrm{~Hz}, \mathrm{~J}_{4,5}=3.5 \mathrm{~Hz}, \mathrm{H}-4\right), 5.03(\mathrm{~s}, \mathrm{br}, 1 \mathrm{H}, \mathrm{H}-1), 4.25\left(\mathrm{dd}, 1 \mathrm{H}, \mathrm{J}_{6 \mathrm{a}, 5}=5.3 \mathrm{~Hz}, \mathrm{~J}_{6 \mathrm{a}, 6 \mathrm{~b}}=\right.$ $12.1 \mathrm{~Hz}, \mathrm{H}-6 \mathrm{a}), 4.09$ (dd, 1H, J $\left.\mathrm{J}_{6 \mathrm{~b}, 5}=2.4 \mathrm{~Hz}, \mathrm{~J}_{6 \mathrm{~b}, 6 \mathrm{a}}=12.1 \mathrm{~Hz}, \mathrm{H}-6 \mathrm{~b}\right), 4.10(\mathrm{~m}, 1 \mathrm{H}, \mathrm{H}-5), 3.96$ (m, $1 \mathrm{H}, \mathrm{H}-7 \mathrm{a}), 3.78$ (ddd, 1H, $\left.\mathrm{J}_{7 \mathrm{~b}, 7 \mathrm{a}}=16.5 \mathrm{~Hz}, \mathrm{~J}_{7 \mathrm{~b}, 8 \mathrm{~b}}=6.8 \mathrm{~Hz}, \mathrm{~J}_{7 \mathrm{~b}, 8 \mathrm{a}}=3.8 \mathrm{~Hz}, \mathrm{H}-7 \mathrm{~b}\right), 2.97$ (t, 2H, $\mathrm{J}_{8,7 \mathrm{a}}$ $\left.=\mathrm{J}_{8,7 \mathrm{~b}}=6.8 \mathrm{~Hz}, 2 \mathrm{H}-8\right), 2.1$ (s, 3H, OAc), 2.07 (s, 3H, OAc). ${ }^{13} \mathrm{C} \mathrm{NMR}\left(50 \mathrm{MHz}, \mathrm{CDCl}_{3}+\mathrm{TMS}\right)$ : 
$\delta 170.7\left(\mathrm{CO}_{2}\right), 170.2\left(\mathrm{CO}_{2}\right), 152.8(\mathrm{C}-9), 141.2(\mathrm{C}-12), 110.3(\mathrm{C}-11), 106.2(\mathrm{C}-10), 130.3(\mathrm{C}-2)$, 126.2 (C-3), 94.1 (C-1), 72.8 (C-5), 66.2 (C-6), 64.3 (C-4), 63.4 (C-7), $28.8(\mathrm{C}-8), 20.9\left(\mathrm{CH}_{3}\right)$, $20.7\left(\mathrm{CH}_{3}\right)$. Anal. calcd for $\mathrm{C}_{16} \mathrm{H}_{20} \mathrm{O}_{7}(\mathrm{M}=324.32)$ : $\mathrm{C}, 59.25 ; \mathrm{H}, 6.22 \%$. Found: $\mathrm{C}, 59.40 ; \mathrm{H}$, $6.16 \%$.

Propyl-(furyl-2')-3', 4,6-di-O-acetyl-2,3-dideoxy- $\alpha$-D-erythro-hex-2-enopyranoside $\quad 3 \boldsymbol{e} \alpha$. Compound $3 \mathbf{e} \alpha$ was obtained as a colourless oil. The specific rotation of pure material is $[\alpha]_{\mathrm{D}}^{20}+75^{\circ}\left(\mathrm{c} 1, \mathrm{CHCl}_{3}\right) ; \mathrm{R}_{\mathrm{f}}=0.46$ (dichloromethane/ethyl acetate 5/0.1 v/v). ${ }^{1} \mathrm{H} \mathrm{NMR}(300$ $\left.\mathrm{MHz}, \mathrm{CDCl}_{3}+\mathrm{TMS}\right): \delta 7.30$ (dd, $\left.1 \mathrm{H}, \mathrm{J}_{13,11}=0.7 \mathrm{~Hz}, \mathrm{~J}_{13,12}=1.9 \mathrm{~Hz}, \mathrm{H}-13\right), 6.27\left(\mathrm{dd}, 1 \mathrm{H}, \mathrm{J}_{12,13}=\right.$ $\left.1.9 \mathrm{~Hz}, \mathrm{~J}_{12,11}=3 \mathrm{~Hz}, \mathrm{H}-12\right), 6.00\left(\mathrm{~d}, 1 \mathrm{H}, \mathrm{J}_{11,12}=3 \mathrm{~Hz}, \mathrm{H}-11\right), 5.96\left(\mathrm{dd}, 1 \mathrm{H}, \mathrm{J}_{2,4}=1.5 \mathrm{~Hz}, \mathrm{~J}_{2,3}=\right.$ $3.3 \mathrm{~Hz}, \mathrm{H}-2), 5.85\left(\mathrm{dd}, 1 \mathrm{H}, \mathrm{J}_{3,4}=1.5 \mathrm{~Hz}, \mathrm{~J}_{3,2}=3.3 \mathrm{~Hz}, \mathrm{H}-3\right), 5.30\left(\mathrm{~d}, \mathrm{br}, 1 \mathrm{H}, \mathrm{J}_{4,5}=1.2 \mathrm{~Hz}, \mathrm{H}-4\right)$, $5.03(\mathrm{~s}, \mathrm{br}, 1 \mathrm{H}, \mathrm{H}-1), 4.25\left(\mathrm{dd}, 1 \mathrm{H}, \mathrm{J}_{6 \mathrm{a}, 5}=5.4 \mathrm{~Hz}, \mathrm{~J}_{6 \mathrm{a}, 6 \mathrm{~b}}=8.3 \mathrm{~Hz}, \mathrm{H}-6 \mathrm{a}\right), 4.18(\mathrm{~m}, 1 \mathrm{H}, \mathrm{H}-$ 6b), 4.08 (m, 1H, H-5), 3.8 (ddd, $\left.1 \mathrm{H}, \mathrm{J}_{7 \mathrm{a}, 8 \mathrm{~b}}=3.2 \mathrm{~Hz}, \mathrm{~J}_{7 \mathrm{a}, 8 \mathrm{a}}=6.3 \mathrm{~Hz}, \mathrm{~J}_{7 \mathrm{a}, 7 \mathrm{~b}}=15.9 \mathrm{~Hz}, \mathrm{H}-7 \mathrm{a}\right), 3.58$ (ddd, $\left.1 \mathrm{H}, \mathrm{J}_{7 \mathrm{~b}, 8 \mathrm{a}}=2.8 \mathrm{~Hz}, \mathrm{~J}_{7 \mathrm{~b}, 8 \mathrm{~b}}=6.2 \mathrm{~Hz}, \mathrm{~J}_{7 \mathrm{~b}, 7 \mathrm{a}}=15.9 \mathrm{~Hz}, \mathrm{H}-7 \mathrm{~b}\right), 2.76\left(\mathrm{t}, 2 \mathrm{H}, \mathrm{J}_{9,8 \mathrm{a}}=\mathrm{J}_{9,8 \mathrm{~b}}=7.5\right.$ $\mathrm{Hz}, 2 \mathrm{H}-9), 1.97$ (m, 2H, 2H-8), 2.1 (s, 3H, OAc), 2.08 (s, 3H, OAc). ${ }^{13} \mathrm{C}$ NMR (50 MHz, $\left.\mathrm{CDCl}_{3}+\mathrm{TMS}\right): \delta 170.7\left(\mathrm{CO}_{2}\right), 170.3\left(\mathrm{CO}_{2}\right), 155.4(\mathrm{C}-10), 110.1(\mathrm{C}-12), 105.0(\mathrm{C}-11), 129.1(\mathrm{C}-$ 2), 127.9 (C-3), 94.5 (C-1), 67.9 (C-5), 67.0 (C-4), 65.3 (C-6), 63.0 (C-7), 28.2 (C-9), 24.7 (C8), $21.0\left(\mathrm{CH}_{3}\right), 20.7\left(\mathrm{CH}_{3}\right)$. Anal. calcd for $\mathrm{C}_{17} \mathrm{H}_{22} \mathrm{O}_{7}(\mathrm{M}=338.35)$ : $\mathrm{C}, 60.34 ; \mathrm{H}, 6.55 \%$. Found: $\mathrm{C}, 60.25 ; \mathrm{H}, 6.60 \%$.

Propyl-(furyl-2')-3', 4,6-di-O-acetyl-2,3-dideoxy- $\beta$-D-erythro-hex-2-enopyranoside $\quad 3 \boldsymbol{e} \beta$. Compound 3e $\beta$ was obtained as a colourless oil. The specific rotation of pure material is $[\alpha]_{\mathrm{D}}^{20}+101^{\mathrm{o}}\left(\mathrm{c} 1, \mathrm{CHCl}_{3}\right) ; \mathrm{R}_{\mathrm{f}}=0.46$ (dichloromethane/ethyl acetate 5/0.1 v/v). ${ }^{1} \mathrm{H}$ NMR $(300$ $\left.\mathrm{MHz}, \mathrm{CDCl}_{3}+\mathrm{TMS}\right): \delta 7.31\left(\mathrm{dd}, 1 \mathrm{H}, \mathrm{J}_{13,11}=0.7 \mathrm{~Hz}, \mathrm{~J}_{13,12}=1.9 \mathrm{~Hz}, \mathrm{H}-13\right), 6.30\left(\mathrm{dd}, 1 \mathrm{H}, \mathrm{J}_{12,13}=\right.$ $\left.1.9 \mathrm{~Hz}, \mathrm{~J}_{12,11}=3 \mathrm{~Hz}, \mathrm{H}-12\right), 6.01\left(\mathrm{~d}, 1 \mathrm{H}, \mathrm{J}_{11,12}=3 \mathrm{~Hz}, \mathrm{H}-11\right), 5.96\left(\mathrm{dd}, 1 \mathrm{H}, \mathrm{J}_{2,4}=1.5 \mathrm{~Hz}, \mathrm{~J}_{2,3}=\right.$ $3.3 \mathrm{~Hz}, \mathrm{H}-2), 5.85$ (dd, $\left.1 \mathrm{H}, \mathrm{J}_{3,4}=1.5 \mathrm{~Hz}, \mathrm{~J}_{3,2}=3.3 \mathrm{~Hz}, \mathrm{H}-3\right), 5.34$ (d, br, $\left.1 \mathrm{H}, \mathrm{J}_{4,5}=1.1 \mathrm{~Hz}, \mathrm{H}-4\right)$, 5.03 (s, br, $1 \mathrm{H}, \mathrm{H}-1), 4.31\left(\mathrm{dd}, 1 \mathrm{H}, \mathrm{J}_{6 \mathrm{a}, 5}=5.4 \mathrm{~Hz}, \mathrm{~J}_{6 \mathrm{a}, 6 \mathrm{~b}}=8.3 \mathrm{~Hz}, \mathrm{H}-6 \mathrm{a}\right), 4.18(\mathrm{~m}, 1 \mathrm{H}, \mathrm{H}-$ 6b), 4.08 (m, 1H, H-5), 3.85 (ddd, $\left.1 \mathrm{H}, \mathrm{J}_{7 \mathrm{a}, 8 \mathrm{~b}}=3.2 \mathrm{~Hz}, \mathrm{~J}_{7 \mathrm{a}, 8 \mathrm{a}}=6.3 \mathrm{~Hz}, \mathrm{~J}_{7 \mathrm{a}, 7 \mathrm{~b}}=15.9 \mathrm{~Hz}, \mathrm{H}-7 \mathrm{a}\right)$, $3.58\left(\mathrm{ddd}, 1 \mathrm{H}, \mathrm{J}_{7 \mathrm{~b}, 8 \mathrm{a}}=2.8 \mathrm{~Hz}, \mathrm{~J}_{7 \mathrm{~b}, 8 \mathrm{~b}}=6.2 \mathrm{~Hz}, \mathrm{~J}_{7 \mathrm{~b}, 7 \mathrm{a}}=15.9 \mathrm{~Hz}, \mathrm{H}-7 \mathrm{~b}\right), 2.72\left(\mathrm{t}, 2 \mathrm{H}, \mathrm{J}_{9,8 \mathrm{a}}=\mathrm{J}_{9,8 \mathrm{~b}}=\right.$ $7.5 \mathrm{~Hz}, 2 \mathrm{H}-9), 1.93$ (m, 2H, $2 \mathrm{H}-8), 2.1$ (s, 3H, OAc), 2.08 (s, 3H, OAc). ${ }^{13} \mathrm{C}$ NMR (50 MHz, $\left.\mathrm{CDCl}_{3}+\mathrm{TMS}\right): \delta 170.7\left(\mathrm{CO}_{2}\right), 170.3\left(\mathrm{CO}_{2}\right), 155.4(\mathrm{C}-10), 110.1(\mathrm{C}-12), 105.0(\mathrm{C}-11), 130.5(\mathrm{C}-$ 2), 126.2 (C-3), 94.3 (C-1), 72.8 (C-5), 67.4 (C-4), 64.4 (C-6), 63.5 (C-7), 28.1 (C-9), 24.6 (C8), $21.0\left(\mathrm{CH}_{3}\right), 20.7\left(\mathrm{CH}_{3}\right)$. Anal. calcd for $\mathrm{C}_{17} \mathrm{H}_{22} \mathrm{O}_{7}(\mathrm{M}=338.35)$ : C, 60.34; $\mathrm{H}, 6.55 \%$. Found: C, $60.10 ; \mathrm{H}, 6.81 \%$.

Furfuryl 4-O-acetyl-2,3-dideoxy- $\alpha$-D-glycero-pent-2-enopyranoside $5 \boldsymbol{a} \alpha$. Compound 5a $\alpha$ was obtained as a clear oil. The specific rotation of pure material is $[\alpha]_{\mathrm{D}}{ }^{20}+104^{\mathrm{o}}\left(\mathrm{c} 1, \mathrm{CHCl}_{3}\right) ; \mathrm{R}_{\mathrm{f}}=$ 0.43 (petroleum ether/ethyl acetate $5 / 2 \mathrm{v} / \mathrm{v}) .{ }^{1} \mathrm{H}$ NMR $\left(200 \mathrm{MHz}, \mathrm{CDCl}_{3}+\mathrm{TMS}\right): \delta 7.43(\mathrm{t}, 1 \mathrm{H}$, $\left.\mathrm{J}_{10,9}=\mathrm{J}_{10,8}=1.4 \mathrm{~Hz}, \mathrm{H}-10\right), 6.36\left(\mathrm{~d}, \mathrm{br}, 2 \mathrm{H}, \mathrm{J}_{9,10}=\mathrm{J}_{8,10}=1.4 \mathrm{~Hz}, \mathrm{H}-8\right.$ and H-9), $5.98\left(\mathrm{dt}, 1 \mathrm{H}, \mathrm{J}_{2,1}\right.$ $\left.=\mathrm{J}_{2,4}=1 \mathrm{~Hz}, \mathrm{~J}_{2,3}=10.3 \mathrm{~Hz}, \mathrm{H}-2\right), 5.83\left(\mathrm{ddd}, 1 \mathrm{H}, \mathrm{J}_{3,1}=2.2 \mathrm{~Hz}, \mathrm{~J}_{3,2}=10.3 \mathrm{~Hz}, \mathrm{~J}_{3,4}=1.6 \mathrm{~Hz}, \mathrm{H}-\right.$ 3), $5.32\left(\mathrm{ddd}, 1 \mathrm{H}, \mathrm{J}_{4,5 \mathrm{a}}=9.8 \mathrm{~Hz}, \mathrm{~J}_{4,3}=1.6 \mathrm{~Hz}, \mathrm{~J}_{4,5 \mathrm{e}}=2 \mathrm{~Hz}, \mathrm{H}-4\right), 5.05$ (s, br, 1H, H-1), 4.72 (d, $\left.1 \mathrm{H}, \mathrm{J}_{6 \mathrm{a}, 6 \mathrm{~b}}=12.8 \mathrm{~Hz}, \mathrm{H}-6 \mathrm{a}\right), 4.56\left(\mathrm{~d}, 1 \mathrm{H}, \mathrm{J}_{6 \mathrm{~b}, 6 \mathrm{a}}=12.8 \mathrm{~Hz}, \mathrm{H}-6 \mathrm{~b}\right), 3.87$ (m, 2H, $\left.2 \mathrm{H}-5\right), 2.07$ (s, $3 \mathrm{H}, \mathrm{OAc}) .{ }^{13} \mathrm{C}$ NMR (50 MHz, $\left.\mathrm{CDCl}_{3}+\mathrm{TMS}\right): \delta 170.3\left(\mathrm{CO}_{2}\right), 151.2(\mathrm{C}-7), 143.0(\mathrm{C}-10), 129.2$ (C-2), 128.8 (C-3), 109.8 (C-9), 109.7 (C-8), 92.9 (C-1), 65.0 (C-5), 63.4 (C-4), 61.6 (C-6), 20.5 $\left(\mathrm{CH}_{3}\right)$. Anal. calcd for $\mathrm{C}_{12} \mathrm{H}_{14} \mathrm{O}_{5}(\mathrm{M}=238.23)$ : C, 60.50; H, 5.92\%. Found: $\mathrm{C}, 60.75 ; \mathrm{H}, 5.80 \%$.

Furfuryl 4-O-acetyl-2,3-dideoxy- $\beta$-D-glycero-pent-2-enopyranoside 5a $\beta$. Compound 5a $\beta$ was obtained as a clear oil. The specific rotation of pure material is $[\alpha]_{\mathrm{D}}{ }^{20}+77^{\circ}\left(\mathrm{c} 1, \mathrm{CHCl}_{3}\right) ; \mathrm{R}_{\mathrm{f}}=$ 0.32 (petroleum ether/ethyl acetate 5/2 v/v). ${ }^{1} \mathrm{H}$ NMR (200 MHz, $\mathrm{CDCl}_{3}+\mathrm{TMS}$ ): $\delta 7.42$ (s, br, $1 \mathrm{H}, \mathrm{H}-10), 6.35\left(\mathrm{dd}, 2 \mathrm{H}, \mathrm{J}_{9,10}=\mathrm{J}_{8,10}=1.1 \mathrm{~Hz}, \mathrm{~J}_{9,8}=3.5 \mathrm{~Hz}, \mathrm{H}-8\right.$ and $\left.\mathrm{H}-9\right), 6.04\left(\mathrm{dd}, 2 \mathrm{H}, \mathrm{J}_{2,1}=\right.$ 
$\mathrm{J}_{3,1}=2.4 \mathrm{~Hz}, \mathrm{~J}_{2,3}=10.2 \mathrm{~Hz}, \mathrm{H}-2$ and H-3), $5.11\left(\mathrm{~d}, 1 \mathrm{H}, \mathrm{J}_{1,2}=2.4 \mathrm{~Hz}, \mathrm{H}-1\right), 4.96\left(\mathrm{t}, \mathrm{br}, 1 \mathrm{H}, \mathrm{J}_{4,5 \mathrm{a}}\right.$ $\left.=\mathrm{J}_{4,3}=2.8 \mathrm{~Hz}, \mathrm{H}-4\right), 4.70\left(\mathrm{~d}, 1 \mathrm{H}, \mathrm{J}_{6 \mathrm{a}, 6 \mathrm{~b}}=12.8 \mathrm{~Hz}, \mathrm{H}-6 \mathrm{a}\right), 4.55\left(\mathrm{~d}, 1 \mathrm{H}, \mathrm{J}_{6 \mathrm{~b}, 6 \mathrm{a}}=12.8 \mathrm{~Hz}, \mathrm{H}-6 \mathrm{~b}\right)$, $4.2\left(\mathrm{dd}, 1 \mathrm{H}, \mathrm{J}_{5 \mathrm{a}, 4}=2.8 \mathrm{~Hz}, \mathrm{~J}_{5 \mathrm{a}, 5 \mathrm{e}}=13.1 \mathrm{~Hz}, \mathrm{H}-5 \mathrm{a}\right), 3.85\left(\mathrm{~d}, 1 \mathrm{H}, \mathrm{J}_{5 \mathrm{e}, 5 \mathrm{a}}=13.1 \mathrm{~Hz}, \mathrm{H}-5 \mathrm{e}\right), 2.10$ (s, $3 \mathrm{H}, \mathrm{OAc}) .{ }^{13} \mathrm{C}$ NMR $\left(50 \mathrm{MHz}, \mathrm{CDCl}_{3}+\mathrm{TMS}\right): \delta 170.7\left(\mathrm{CO}_{2}\right), 151.1$ (C-7), 143.1 (C-10), 130.7 (C-2), 125.2 (C-3), 110.4 (C-9), 109.8 (C-8), 91.7 (C-1), 65.0 (C-5), 63.3 (C-4), 61.4 (C-6), 20.6 $\left(\mathrm{CH}_{3}\right)$. Anal. calcd for $\mathrm{C}_{12} \mathrm{H}_{14} \mathrm{O}_{5}(\mathrm{M}=238.23)$ : C, 60.50; H, 5.92\%. Found: $\mathrm{C}, 60.91 ; \mathrm{H}, 5.75 \%$.

Ethyl-(furyl-2')-2'" 4-O-acetyl-2,3-dideoxy- $\alpha$-D-glycero-pent-2-enopyranoside $5 d \alpha$. Compound $5 d \alpha$ was obtained as a clear oil. The specific rotation of pure material is $[\alpha]_{\mathrm{D}}{ }^{20}+20^{\circ}(\mathrm{c} 0.25$, $\left.\mathrm{CHCl}_{3}\right) ; \mathrm{R}_{\mathrm{f}}=0.44$ (petroleum ether/ethyl acetate $\left.5 / 2 \mathrm{v} / \mathrm{v}\right) .{ }^{1} \mathrm{H}$ NMR $\left(200 \mathrm{MHz}, \mathrm{CDCl}_{3}+\mathrm{TMS}\right): \delta$ 7.30 (s, br, 1H, H-11), 6.30 (dd, 1H, $\left.\mathrm{J}_{10,11}=1.5 \mathrm{~Hz}, \mathrm{~J}_{10,9}=3 \mathrm{~Hz}, \mathrm{H}-10\right), 6.09$ (d, 1H, J ${ }_{9,10}=3 \mathrm{~Hz}$, H-9), 5.94 (d, br, 1H, J $\left.{ }_{2,3}=10.4 \mathrm{~Hz}, \mathrm{H}-2\right), 5.83\left(\mathrm{dd}, 1 \mathrm{H}, \mathrm{J}_{3,4}=1.6 \mathrm{~Hz}, \mathrm{~J}_{3,2}=10.4 \mathrm{~Hz}, \mathrm{H}-3\right), 5.28$ $\left(\mathrm{ddd}, 1 \mathrm{H}, \mathrm{J}_{4,3}=1.6 \mathrm{~Hz}, \mathrm{~J}_{4,5 \mathrm{e}}=1.7 \mathrm{~Hz}, \mathrm{~J}_{4,5 \mathrm{a}}=5.8 \mathrm{~Hz}, \mathrm{H}-4\right), 4.95(\mathrm{~s}, \mathrm{br}, 1 \mathrm{H}, \mathrm{H}-1), 4.05(\mathrm{dd}, 1 \mathrm{H}$, $\left.\mathrm{J}_{5 \mathrm{e}, 4}=1.7 \mathrm{~Hz}, \mathrm{~J}_{5 \mathrm{e}, 5 \mathrm{a}}=16.5 \mathrm{~Hz}, \mathrm{H}-5 \mathrm{e}\right), 3.81\left(\mathrm{~m}, 3 \mathrm{H}, \mathrm{J}_{5 \mathrm{a}, 4}=5.8 \mathrm{~Hz}, \mathrm{~J}_{6 \mathrm{a}, 6 \mathrm{~b}}=11.1 \mathrm{~Hz}, \mathrm{~J}_{5 \mathrm{a}, 5 \mathrm{e}}=16.5\right.$ $\mathrm{Hz}, \mathrm{H}-5 \mathrm{a}, 2 \mathrm{H}-6), 2.78$ (t, 2H, $\left.\mathrm{J}_{7,6 \mathrm{a}}=\mathrm{J}_{7,6 \mathrm{~b}}=6.9 \mathrm{~Hz}, 2 \mathrm{H}-7\right), 2.10$ (s, 3H, OAc). ${ }^{13} \mathrm{C}$ NMR (50 $\left.\mathrm{MHz}, \mathrm{CDCl}_{3}+\mathrm{TMS}\right): \delta 170.5\left(\mathrm{CO}_{2}\right), 152.8(\mathrm{C}-8), 141.2$ (C-11), 129.1 (C-2), $129.0(\mathrm{C}-3), 110.3$ (C-10), 106.0 (C-9), 94.4 (C-1), 66.7 (C-5), 65.0 (C-4), 60.3 (C-6), 28.9 (C-7), $21.0\left(\mathrm{CH}_{3}\right)$. Anal. calcd for $\mathrm{C}_{13} \mathrm{H}_{16} \mathrm{O}_{5}(\mathrm{M}=252.26)$ : $\mathrm{C}, 61.89 ; \mathrm{H}, 6.39 \%$. Found: $\mathrm{C}, 62.15 ; \mathrm{H}, 6.27 \%$.

Ethyl-(furyl-2')-2"' 4-O-acetyl-2,3-dideoxy- $\beta$-D-glycero-pent-2-enopyranoside $5 \boldsymbol{d} \beta$. Compound 5d $\beta$ was obtained as a clear oil. The specific rotation of pure material is $[\alpha]_{D}{ }^{20}+108^{\circ}$ (c 1 , $\left.\mathrm{CHCl}_{3}\right) ; \mathrm{R}_{\mathrm{f}}=0.35$ (petroleum ether/ethyl acetate $5 / 2 \mathrm{v} / \mathrm{v}$ ). ${ }^{1} \mathrm{H}$ NMR $\left(200 \mathrm{MHz}, \mathrm{CDCl}_{3}+\mathrm{TMS}\right): \delta$ $7.30\left(\mathrm{dd}, 1 \mathrm{H}, \mathrm{J}_{11,9}=0.8 \mathrm{~Hz}, \mathrm{~J}_{11,10}=1.8 \mathrm{~Hz}, \mathrm{H}-11\right), 6.29\left(\mathrm{dd}, 1 \mathrm{H}, \mathrm{J}_{10,11}=1.8 \mathrm{~Hz}, \mathrm{~J}_{10,9}=3.2 \mathrm{~Hz}\right.$, $\mathrm{H}-10), 6.07\left(\mathrm{dd}, 1 \mathrm{H}, \mathrm{J}_{9,11}=0.8 \mathrm{~Hz}, \mathrm{~J}_{9,10}=3.2 \mathrm{~Hz}, \mathrm{H}-9\right), 6.02\left(\mathrm{dd}, 2 \mathrm{H}, \mathrm{J}_{3,1}=\mathrm{J}_{2,1}=2.6 \mathrm{~Hz}, \mathrm{~J}_{2,3}=\right.$ $10.1 \mathrm{~Hz}, \mathrm{H}-2$ and H-3), $5.00\left(\mathrm{t}, 1 \mathrm{H}, \mathrm{J}_{1,2}=\mathrm{J}_{1,3}=2.6 \mathrm{~Hz}, \mathrm{H}-1\right), 4.93\left(\mathrm{dd}, 1 \mathrm{H}, \mathrm{J}_{4,5 \mathrm{e}}=1.7 \mathrm{~Hz}, \mathrm{~J}_{4,5 \mathrm{a}}=\right.$ $2.7 \mathrm{~Hz}, \mathrm{H}-4), 4.11$ (dd, $\left.1 \mathrm{H}, \mathrm{J}_{5 \mathrm{a}, 4}=2.7 \mathrm{~Hz}, \mathrm{~J}_{5 \mathrm{a}, 5 \mathrm{e}}=16.1 \mathrm{~Hz}, \mathrm{H}-5 \mathrm{a}\right), 3.99$ (dd, $1 \mathrm{H}, \mathrm{J}_{6 \mathrm{a}, 7}=6.9 \mathrm{~Hz}$, $\left.\mathrm{J}_{6 \mathrm{a}, 6 \mathrm{~b}}=9.9 \mathrm{~Hz}, \mathrm{H}-6 \mathrm{a}\right), 3.82\left(\mathrm{dd}, 1 \mathrm{H}, \mathrm{J}_{6 \mathrm{~b}, 7}=6.9 \mathrm{~Hz}, \mathrm{~J}_{6 \mathrm{~b}, 6 \mathrm{a}}=9.9 \mathrm{~Hz}, \mathrm{H}-6 \mathrm{~b}\right), 3.80\left(\mathrm{~m}, 1 \mathrm{H}, \mathrm{J}_{5 \mathrm{e}, 4}=1.7\right.$ $\left.\mathrm{Hz}, \mathrm{J}_{5 \mathrm{e}, 5 \mathrm{a}}=16.1 \mathrm{~Hz}, \mathrm{H}-5 \mathrm{e}\right), 2.98\left(\mathrm{t}, 2 \mathrm{H}, \mathrm{J}_{7,6 \mathrm{a}}=\mathrm{J}_{7,6 \mathrm{~b}}=6.8 \mathrm{~Hz}, 2 \mathrm{H}-7\right), 2.10(\mathrm{~s}, 3 \mathrm{H}, \mathrm{OAc}) .{ }^{13} \mathrm{C}$ NMR (50 MHz, CDCl $\left.{ }_{3}+\mathrm{TMS}\right): \delta 170.6\left(\mathrm{CO}_{2}\right), 152.8$ (C-8), 141.2 (C-11), 130.8 (C-2), 125.1 (C-3), 110.3 (C-10), 106.1 (C-9), 93.1 (C-1), 66.5 (C-5), 64.2 (C-4), 61.3 (C-6), 29.0 (C-7), 21.1 $\left(\mathrm{CH}_{3}\right)$. Anal. calcd for $\mathrm{C}_{13} \mathrm{H}_{16} \mathrm{O}_{5}(\mathrm{M}=252.26)$ : $\mathrm{C}, 61.89 ; \mathrm{H}, 6.39 \%$. Found: $\mathrm{C}, 61.53 ; \mathrm{H}, 6.51 \%$.

Propyl-(furyl-2')-3', 4-O-acetyl-2,3-dideoxy- $\alpha$-D-glycero-pent-2-enopyranoside $\quad 5 \boldsymbol{e} \quad \alpha$. Compound 5e $\alpha$ was obtained as a clear oil. The specific rotation of pure material is $[\alpha]_{\mathrm{D}}^{20}+59^{\circ}\left(\mathrm{c} 0.78, \mathrm{CHCl}_{3}\right) ; \mathrm{R}_{\mathrm{f}}=0.31$ (petroleum ether/ethyl acetate $\left.5 / 0.5 \mathrm{v} / \mathrm{v}\right) .{ }^{1} \mathrm{H}$ NMR $(200$ $\left.\mathrm{MHz}, \mathrm{CDCl}_{3}+\mathrm{TMS}\right): \delta 7.30\left(\mathrm{dd}, 1 \mathrm{H}, \mathrm{J}_{12,10}=0.7 \mathrm{~Hz}, \mathrm{~J}_{12,11}=1.8 \mathrm{~Hz}, \mathrm{H}-12\right), 6.28\left(\mathrm{dd}, 1 \mathrm{H}, \mathrm{J}_{11,12}=\right.$ $\left.1.8 \mathrm{~Hz}, \mathrm{~J}_{11,10}=3.1 \mathrm{~Hz}, \mathrm{H}-11\right), 6.00\left(\mathrm{dd}, 1 \mathrm{H}, \mathrm{J}_{10,12}=0.7 \mathrm{~Hz}, \mathrm{~J}_{10,11}=3.1 \mathrm{~Hz}, \mathrm{H}-10\right), 5.90(\mathrm{dd}, 1 \mathrm{H}$, $\left.\mathrm{J}_{2,1}=0.6 \mathrm{~Hz}, \mathrm{~J}_{2,3}=9.1 \mathrm{~Hz}, \mathrm{H}-2\right), 5.84\left(\mathrm{dd}, 1 \mathrm{H}, \mathrm{J}_{3,4}=2.1 \mathrm{~Hz}, \mathrm{~J}_{3,2}=9.1 \mathrm{~Hz}, \mathrm{H}-3\right), 5.28(\mathrm{ddd}, 1 \mathrm{H}$, $\left.\mathrm{J}_{4,3}=2.1 \mathrm{~Hz}, \mathrm{~J}_{4,5 \mathrm{e}}=4.1 \mathrm{~Hz}, \mathrm{~J}_{4,5 \mathrm{a}}=7.7 \mathrm{~Hz}, \mathrm{H}-4\right), 4.90(\mathrm{~s}, \mathrm{br}, 1 \mathrm{H}, \mathrm{H}-1), 3.89$ (dd, $1 \mathrm{H}, \mathrm{J}_{5 \mathrm{e}, 4}=4.1$ $\left.\mathrm{Hz}, \mathrm{J}_{5 \mathrm{e}, 5 \mathrm{a}}=16.5 \mathrm{~Hz}, \mathrm{H}-5 \mathrm{e}\right), 3.84\left(\mathrm{dd}, 1 \mathrm{H}, \mathrm{J}_{5 \mathrm{a}, 4}=7.7 \mathrm{~Hz}, \mathrm{~J}_{5 \mathrm{a}, 5 \mathrm{e}}=16.5 \mathrm{~Hz}, \mathrm{H}-5 \mathrm{a}\right), 3.79(\mathrm{~m}, 1 \mathrm{H}, \mathrm{H}-$ 6a), $3.5\left(\mathrm{ddd}, 1 \mathrm{H}, \mathrm{J}_{6 \mathrm{~b}, 7 \mathrm{a}}=3.0 \mathrm{~Hz}, \mathrm{~J}_{6 \mathrm{~b}, 7 \mathrm{~b}}=6.4 \mathrm{~Hz}, \mathrm{~J}_{6 \mathrm{~b}, 6 \mathrm{a}}=16.0 \mathrm{~Hz}, \mathrm{H}-6 \mathrm{~b}\right), 2.80\left(\mathrm{t}, 2 \mathrm{H}, \mathrm{J}_{8,7 \mathrm{a}}=\mathrm{J}_{8,7 \mathrm{~b}}=\right.$ $7.5 \mathrm{~Hz}, 2 \mathrm{H}-8), 2.10$ (s, 3H, OAc), 1.97 (m, 2H, $2 \mathrm{H}-7$ ). ${ }^{13} \mathrm{C} \mathrm{NMR}\left(50 \mathrm{MHz}, \mathrm{CDCl}_{3}+\mathrm{TMS}\right): \delta$ $170.4\left(\mathrm{CO}_{2}\right), 155.5$ (C-9), 140.0 (C-12), 129.3 (C-2), 128.8 (C-3), 110.2 (C-11), 105.1 (C-10), 94.3 (C-1), 67.7 (C-5), 65.0 (C-4), $60.1(\mathrm{C}-6), 28.2(\mathrm{C}-8), 24.6(\mathrm{C}-7), 21.0\left(\mathrm{CH}_{3}\right)$. Anal. calcd for $\mathrm{C}_{14} \mathrm{H}_{18} \mathrm{O}_{5}(\mathrm{M}=266.28): \mathrm{C}, 63.14 ; \mathrm{H}, 6.81 \%$. Found: $\mathrm{C}, 62.98 ; \mathrm{H}, 6.88 \%$.

Propyl-(furyl-2')-3', $\quad 4$-O-acetyl-2,3-dideoxy- $\beta$-D-glycero-pent-2-enopyranoside $\quad \mathbf{5 e} \quad \beta$. Compound $5 \mathbf{e} \beta$ was obtained as a clear oil. The specific rotation of pure material is $[\alpha]_{\mathrm{D}}{ }^{20}+100^{\circ}$ (c $1, \mathrm{CHCl}_{3}$ ); $\mathrm{R}_{\mathrm{f}}=0.26$ (petroleum ether/ethyl acetate 5/0.5 v/v). ${ }^{1} \mathrm{H} \mathrm{NMR}(200 \mathrm{MHz}$, $\mathrm{CDCl}_{3}+\mathrm{TMS}$ ): $\delta 7.30$ (s, br, $\left.1 \mathrm{H}, \mathrm{H}-12\right), 6.27$ (dd, 1H, $\left.\mathrm{J}_{11,12}=1.9 \mathrm{~Hz}, \mathrm{~J}_{11,10}=3.0 \mathrm{~Hz}, \mathrm{H}-11\right), 6.07$ 
$\left(\mathrm{d}, 1 \mathrm{H}, \mathrm{J}_{10,11}=3 \mathrm{~Hz}, \mathrm{H}-10\right), 6.00\left(\mathrm{dd}, 2 \mathrm{H}, \mathrm{J}_{2,1}=4.1 \mathrm{~Hz}, \mathrm{~J}_{2,3}=\mathrm{J}_{3,2}=10 \mathrm{~Hz}, \mathrm{H}-2\right.$ and H-3), $4.98(\mathrm{~s}$, br, 1H, H-1), 4.93 (m, 1H, H-4), 4.20 (dd, 1H, J $\left.5_{5,4}=2.8 \mathrm{~Hz}, \mathrm{~J}_{5 \mathrm{a}, 5 \mathrm{e}}=13 \mathrm{~Hz}, \mathrm{H}-5 \mathrm{a}\right), 3.84$ (d, br, $\left.1 \mathrm{H}, \mathrm{J}_{5 \mathrm{e}, 5 \mathrm{a}}=13 \mathrm{~Hz}, \mathrm{H}-5 \mathrm{e}\right), 3.82(\mathrm{~m}, 1 \mathrm{H}, \mathrm{H}-6 \mathrm{a}), 3.50\left(\mathrm{ddd}, 1 \mathrm{H}, \mathrm{J}_{6 \mathrm{~b}, 7 \mathrm{a}}=3 \mathrm{~Hz}, \mathrm{~J}_{6 \mathrm{~b}, 7 \mathrm{~b}}=6.3 \mathrm{~Hz}, \mathrm{~J}_{6 \mathrm{~b}, 6 \mathrm{a}}=\right.$ $16 \mathrm{~Hz}, \mathrm{H}-6 \mathrm{~b}), 2.72$ (t, 2H, $\left.\mathrm{J}_{8,7 \mathrm{a}}=\mathrm{J}_{8,7 \mathrm{~b}}=7.4 \mathrm{~Hz}, 2 \mathrm{H}-8\right), 2.10$ (s, 3H, OAc), 1.97 (m, 2H, $2 \mathrm{H}-7$ ).

${ }^{13} \mathrm{C}$ NMR (50 MHz, CDCl $\left.{ }_{3}+\mathrm{TMS}\right): \delta 170.5\left(\mathrm{CO}_{2}\right), 155.4(\mathrm{C}-9), 140.9(\mathrm{C}-12), 130.9(\mathrm{C}-2)$, 124.9 (C-3), 110.1 (C-11), 105.0 (C-10), 93.1 (C-1), 67.5 (C-5), 63.4 (C-4), 61.2 (C-6), 28.2 (C8), 24.6 (C-7), $20.9\left(\mathrm{CH}_{3}\right)$. Anal. calcd for $\mathrm{C}_{14} \mathrm{H}_{18} \mathrm{O}_{5}(\mathrm{M}=266.28)$ : C, 63.14; $\mathrm{H}, 6.81 \%$. Found: $\mathrm{C}, 63.08 ; \mathrm{H}, 6.94 \%$.

\section{ACKNOWLEDMENT}

This work was supported by CNRS (Centre National de la Recherche Scientifique) and MNERT (Ministère de l'Education Nationale de la Recherche et de la Technologie), France.

\section{REFERENCES}

1. Collins, P.M.; Ferrier, R.J. Monosaccharides, Their Chemistry and Their Roles in Natural Products, John Wiley: Chichester, U.K.; 1995; pp 317-326.

2. Danishefsky, S.J.; Bilodeau, M.T. Angew. Chem. Int. Ed. Engl. 1996, 35, 1380.

3. Izumi, M.; Ichikawa, Y. Tetrahedron Lett. 1998, 39, 2079.

4. Williams, N.R.; Wander, J.D. The Carbohydrate Chemistry and Biochemistry, Academic Press: New York; 1980; p 761.

5. Kagasaki, T.; Hosoya, T.; Takahashi, S. J. Antibiot. 1993, 46, 1643.

6. Takahashi, S.; Kinoshita, T.; Takahashi, M. J. Antibiot. 1994, 47, 95.

7. Ferrier, R.J.; Prasad, N. J. Chem. Soc. C 1969, 570.

8. Nair, V.; George, T.G.; Nair, L.G.; Panicker, S.B. Tetrahedron Lett. 1999, 40, 1195.

9. Paul, S.; Jayaraman, N. Carbohydr. Res. 2004, 339, 2197.

10. Yadav, J.S.; Subba Reddy, B.V.; Pandey, S.K. New J. Chem. 2001, 25, 538.

11. De Freitas Filho, J.R.; Srivastava, R.M.; Soro, Y.; Cottier, L.; Descotes, G. J. Carbohydr. Chem. 2001, 20, 561.

12. Harmata, M.; Gamlath, C.B.; Barnes, C.L.; Jones, D.E. J. Org. Chem. 1995, 60, 5077.

13. De Shong, P.; Waltermire, R.E.; Ammon, H.L. J. Am. Chem. Soc. 1998, 110, 1901.

14. Shostakovskii, M.F.; Annenkova, V.M.; Gaistseva, E.A.; Lavrova, K.F.; Polyakov, A.I. Izvest. Sibirsk. Otdel. Akad. Nauk. S.S.S.R., Ser. Khim. Nauk. 1967, 163.

15. Doboszewski, B.; Blaton, N.; Herdewijn, P. J. Org. Chem. 1995, 60, 7909.

16. Ferrier, R.J.; Sankey, G.H. J. Chem. Soc. C 1968, 974. 